\title{
Two-Photon Absorption of Nonclassical Light
}

\author{
J. Gea-Banacloche \\ Instituto de Optica "Daza de Valdés," Consejo Superior de Investigaciones Científicas, Serrano 121, 28006-Madrid, Spain
}

(Received 29 December 1988)

\begin{abstract}
Two-photon absorption of light with nonclassical (squeezed) fluctuations is considered. Several unique effects are predicted for various possible regimes. These include a linear (instead of quadratic) growth of the absorption rate with the light intensity for weak fields, the possibility of a decreasing absorption rate with increasing intensity, and orders-of-magnitude differences between absorption rates for phase- and amplitude-squeezed beams of the same intensity.
\end{abstract}

PACS numbers: $42.50 . \mathrm{Dv}, 32.80 \mathrm{Wr}, 42.50 . \mathrm{Kb}$

Multiphoton absorption processes are known to be very sensitive to the fluctuations of the incident light, since they probe higher-order correlation functions of the field than the more common one-photon processes in terms of which the intensity and the spectrum (firstorder correlation functions) are usually defined. ${ }^{1-4}$ It is a natural question to ask about the influence of nonclassical fluctuations (in particular, squeezed fluctuations) in such processes, now that sources of squeezed light have become available. ${ }^{5}$ The close ties between squeezed states and two-photon processes ${ }^{6,7}$ suggests that twophoton absorption might be especially sensitive to the unique properties of squeezed light. The results to be presented here indicate that such is indeed the case.

Recent studies of unusual effects in the interaction of squeezed light with atomic systems have included suppressed spontaneous decay, ${ }^{8}$ and subnatural linewidths in resonance fluorescence ${ }^{9}$ and (one-photon) absorption, ${ }^{10}$ among others. In these papers all the modes with which the atom might interact (in particular, all the possible decay modes) are assumed to be squeezed. The possibility of observing similar, if less dramatic, effects under less restrictive conditions has been pointed out recently. ${ }^{11}$ One may also note that in lasers, in particular, it might be possible to observe interesting effects through the squeezing of only a few modes. ${ }^{12,13}$

The system studied here is simpler than most of the above (conceptually, at least) in that only one mode of the field is assumed to be squeezed. To be specific, we consider an atom placed in a beam of squeezed light which is near resonant with a two-photon transition. The other modes of the field are in the ordinary vacuum state, and no subnatural linewidths are obtained (to second order in the interaction). Nevertheless, the results are unique enough to deserve notice.

Mollow' derived the following expression for the twophoton absorption rate from a stationary field through second order in perturbation theory:

$$
w_{2}=2|g|^{2} \int_{-\infty}^{\infty} d t e^{2 i \omega_{0} t-\Gamma|t|} G^{(2)}(-t,-t ; t, t) .
$$

Here $\Gamma$ is the width of the upper atomic level, $\omega_{0}$ is the atomic frequency for the two-photon transition, $g$ is a coupling constant, and the second-order correlation function $G^{(2)}$ is defined in the standard way,

$$
\begin{aligned}
G^{(2)} & \left(t_{1}, t_{2} ; t_{3}, t_{4}\right) \\
& =\left\langle E^{(-)}\left(t_{1}\right) E^{(-)}\left(t_{2}\right) E^{(+)}\left(t_{3}\right) E^{(+)}\left(t_{4}\right)\right\rangle .
\end{aligned}
$$

The time arguments appearing in (1) make this a different second-order correlation function from the well-known intensity correlation function (see Ref. 14 for a discussion of this point).

The predictions from Eq. (1) for several kinds of thermal and laser fields have been confirmed experimentally. $^{2}$ To study the predictions for squeezed states, it is useful to consider two separate limits.

(i) Short-lived atoms. - If the atomic lifetime is much shorter than any of the coherence times characteristic of the squeezed light, the absorption spectrum will be approximately given by

$$
w_{2}(\omega)=2|g|^{2} G^{(2)}(0) \frac{\Gamma / 2}{(\Gamma / 2)^{2}+\left(2 \omega-\omega_{0}\right)^{2}},
$$

where $\omega$ is the laser frequency. In this case the absorption rate depends on intensity fluctuations only ("bunching" or "antibunching"), as given by $G^{(2)}(0)$, which is the correlation function (2) with all its arguments equal. The photon statistics of squeezed light have been extensively studied. ${ }^{15,16}$

Consider an ideal squeezed state consisting of a coherent component of intensity $I_{c}$ and a "squeezedvacuum" part of intensity $I_{\text {sv }}$. For the squeezed-vacuum part we may take the light emitted by a degenerate optical-parametric oscillator (OPO) below threshold. ${ }^{17,18}$ This system has two parameters, the (intensity) cavity loss rate $\gamma$ and the ratio of pump amplitude to threshold-pump amplitude which in the notation of Ref. 18 is equal to $2 \epsilon / \gamma$. The output intensity is given by

$$
I_{\mathrm{sv}}=2 \gamma \epsilon^{2} /\left(\gamma^{2}-4 \epsilon^{2}\right) \text {. }
$$

In terms of $I_{c}, I_{\mathrm{sv}}$, and $\gamma, G^{(2)}(0)$ is given by

$G^{(2)}(0)=I_{c}^{2}+I_{c}\left[4 I_{\mathrm{sv}} \pm\left(4 I_{\mathrm{sv}}^{2}+2 \gamma I_{\mathrm{sv}}\right)^{1 / 2}\right]+3 I_{\mathrm{sv}}^{2}+\frac{1}{2} \gamma I_{\mathrm{sv}}$, 
where the plus and minus signs hold for phase- and amplitude-squeezed light, respectively.

Perhaps the most remarkable feature of Eq. (5) is that when $I_{c}=0$ (pure squeezed vacuum) it grows as $I_{\mathrm{sv}}$ for weak fields. That is, by Eq. (3), the two-photon absorption rate grows linearly with the field intensity for weak squeezed-vacuum light. While it may be argued that this is not necessarily a nonclassical effect [no classical inequalities are in fact violated by Eq. (5) when $I_{c}=0$ ], it certainly is a sharp departure from the behavior of all known classical fields (such as thermal, or coherent, or superpositions of both) for which the two-photon absorption rate is, of course, quadratic in the intensity for low intensities.

The precise condition for the linear term to be larger than the quadratic in (5) is

$$
4 \epsilon<\gamma
$$

as is easily obtained from (4). The value $4 \epsilon=\gamma$ already corresponds to a field which is squeezed $90 \%$ below the vacuum level, so (6) is not a very restrictive condition; the experiments reported in Ref. 17 did not go much beyond this range of values. Still, it is only fair to say that these are very weak fields. The condition $\gamma \ll \Gamma$ implies, in fact, that the atom sees on the average less than one photon during its lifetime (which, in passing, may help explain the unusual effect and also appears to suggest that the quantum nature of light, in particular its discreteness, is necessary to its understanding). This does not necessarily place the effect beyond the experimental reach: One may recall, for instance, that Rabi oscillations for very few photons have already been observed in the one-photon micromaser, ${ }^{19}$ and a two- photon micromaser has already been demonstrated. ${ }^{20}$

Turning now to the case when the coherent component $I_{c}$ is nonzero, one finds that, also when condition (6) holds, the term in square brackets in Eq. (5) is negative for amplitude-squeezed light. This means that for fixed $I_{\mathrm{sv}}$ one may have a decreasing absorption rate for increasing $I_{c}$; i.e., the absorption rate decreases even as the total intensity $I_{c}+I_{\text {sv }}$ increases. This effect is related to the fact that as $I_{c}$ increases from zero, the amplitudesqueezed light goes from being bunched to being antibunched; since the atom needs to absorb two photons simultaneously, the antibunching of light may lead to a decrease in the absorption rate, at least for $I_{c}$ not too large.

The same behavior is observed if $I_{\mathrm{sv}}$ is increased from zero for fixed $I_{c}$ (one could think of starting with a coherent field and then squeezing its amplitude fluctuations); even though the total intensity increases with the squeezing, the total absorption rate is easily seen to decrease initially. There are, of course, explicitly nonclassical effects, since they involve antibunching. Note also that this last effect is not necessarily restricted to very weak fields, since the coherent component could be large.

The difference between the absorption rates for amplitude- and phase-squeezed light may be even more dramatic in the case of long-lived atoms, considered next.

(ii) Long-lived atoms. - The (first-order) spectrum of the OPO light has the two characteristic decay rates $\gamma \pm 2 \epsilon .{ }^{18}$ When the atomic decay rate is not much larger than these, the full integral in (1) must be evaluated.

Allowing for the possibility of phase diffusion of the coherent amplitude, the correlation function in (1) breaks up into

$$
\begin{aligned}
G^{(2)}(-t,-t ; t, t)=I_{c}^{2} e^{-4 i \omega t}\left\langle e^{-2 i\left[\phi_{c}(t)-\phi_{c}(-t)\right]}\right\rangle & \\
& -I_{c} e^{-2 i \omega t}\left[\left\langle e^{2 i \phi_{c}(-t)} E_{\mathrm{sv}}^{(+)}(t) E_{\mathrm{sv}}^{(+)}(t)\right\rangle\right. \\
& +\left\langle e^{-2 i \phi_{c}(t)} E_{\mathrm{sv}}^{(-)}(-t) E_{\mathrm{sv}}^{(-)}(-t)\right\rangle \\
& \left.+2\left\langle e^{-i\left[\phi_{c}(t)-\phi_{c}(-t)\right]} E_{\mathrm{sv}}^{(-)}(-t) E_{\mathrm{sv}}^{(+)}(t)\right\rangle\right] \\
& +\left\langle E_{\mathrm{sv}}^{(-)}(-t) E_{\mathrm{sv}}^{(-)}(-t) E_{\mathrm{sv}}^{(+)}(t) E_{\mathrm{sv}}^{(+)}(t)\right\rangle .
\end{aligned}
$$

If the expectation value $\left\langle E_{\mathrm{sv}}^{(+)}(t) E_{\mathrm{sv}}^{(+)}(t)\right\rangle$ is calculated using the expressions available in the literature, ${ }^{18}$ it appears to be time independent. In reality, however, such terms should eventually decay to zero, due to phase diffusion of the pump beam which causes the phase about which one "squeezes" to drift. If both the OPO pump and the coherent component are assumed to diffuse in phase independently, at rates $D_{p}$ and $D_{c}$, say, the first two terms in square brackets in (7) average to zero in the long-time limit; in fact, for the correlation function to be stationary such terms should not be included in (7) in that case (rather, they should be set equal to zero from the start).
For these terms to contribute to a truly stationary process, the two phases involved (OPO and coherent component) must be correlated. This may be the case if the coherent component has been obtained from the same pump beam through subharmonic generation. In this case the phase-sensitive terms may remain in (7), although they need to be reevaluated according to the assumption of correlated phase diffusion. ${ }^{21}$

This is not necessary, however, if $D_{p}, D_{c} \ll \Gamma$. Then to evaluate the integral (1) these terms may be taken as time independent. In this limit (but not, in general, when the pump phase diffusion is important) the last 
term in (7) factors in the manner characteristic of Gaussian noise ${ }^{7,18}$ :

$$
\begin{aligned}
\left\langle E_{\mathrm{sv}}^{(-)}(-t) E_{\mathrm{sv}}^{(-)}(-t) E_{\mathrm{sv}}^{(+)}(t) E_{\mathrm{sv}}^{(+)}(t)\right\rangle=\left\langle E_{\mathrm{sv}}^{(-)}(-t) E_{\mathrm{sv}}^{(-)}(-t)\right\rangle\left\langle E_{\mathrm{sv}}^{(+)}(t) E_{\mathrm{sv}}^{(+)}(t)\right\rangle \\
+2\left\langle E_{\mathrm{sv}}^{(-)}(-t) E_{\mathrm{sv}}^{(+)}(t)\right\rangle\left\langle E_{\mathrm{sv}}^{(-)}(-t) E_{\mathrm{sv}}^{(+)}(t)\right\rangle
\end{aligned}
$$

and $\phi_{c}$ may be treated as a constant. The correlation functions appearing in (7) and (8) may be found, for instance, in Ref. 17.

The main features of the absorption spectrum $w_{2}(\omega)$ in the limit $D_{p}, D_{c} \ll \Gamma \ll \gamma \pm 2 \epsilon$ are the following. The spectrum is given by a superposition of Lorentzians of which the leading are the narrowest, which have the width of the atomic transition $(\Gamma)$. Their origin is precisely the phase-sensitive part of the squeezed fluctuations (the terms $\left\langle E_{\mathrm{sv}}^{(+)} E_{\mathrm{sv}}^{(+)}\right\rangle$and $\left\langle E_{\mathrm{sv}}^{(-)} E_{\mathrm{sv}}^{(-)}\right\rangle$just discussed), and the coherent component. Explicitly, we have

$$
w_{2}(\omega)=2|g|{ }^{2}\left[I_{c} \pm\left(I_{\mathrm{sv}}^{2}+\gamma I_{\mathrm{sv}} / 2\right)^{1 / 2}\right] \frac{\Gamma / 2}{(\Gamma / 2)^{2}+\left(2 \omega-\omega_{0}\right)^{2}}+\cdots .
$$

Equation (8) implies that for $I_{c}=\left(I_{\mathrm{sv}}^{2}+\gamma I_{\mathrm{sv}} / 2\right)^{1 / 2}$ the leading terms in the absorption spectrum can be made to vanish for amplitude-squeezed light. The next largest terms in that case are much broader (of width $\sim \gamma-2 \epsilon$ ) and correspondingly lower [by the ratio $\Gamma /(\gamma-2 \epsilon)$ ]. We thus have the possibility of having a difference of perhaps orders of magnitude between the absorption rates for phase- and amplitude-squeezed light of the same intensity.

This point is illustrated in Fig. 1 where $\epsilon=0.1 \gamma$ and $\Gamma=0.1 \gamma$ (and the value of $I_{c}$ indicated above) have been chosen. The actual degree of squeezing for this set of parameters is rather modest (about 56\%). The amplitude-squeezed beam is also antibunched $\left[g^{(2)}(0)=0.6\right]$. The phase-squeezed beam is bunched $\left[g^{(2)}(0)=3.4\right]$. The absorption rate for a pure coherent beam with the same $I_{c}$ has also been plotted for reference. The total intensity in either of the two squeezed beams is only about $20 \%$ larger than $I_{c}$.

Finally, the case when $\Gamma \ll D_{p}, D_{c} \ll \gamma \pm 2 \epsilon$ and the phases of pump and coherent component diffuse in a

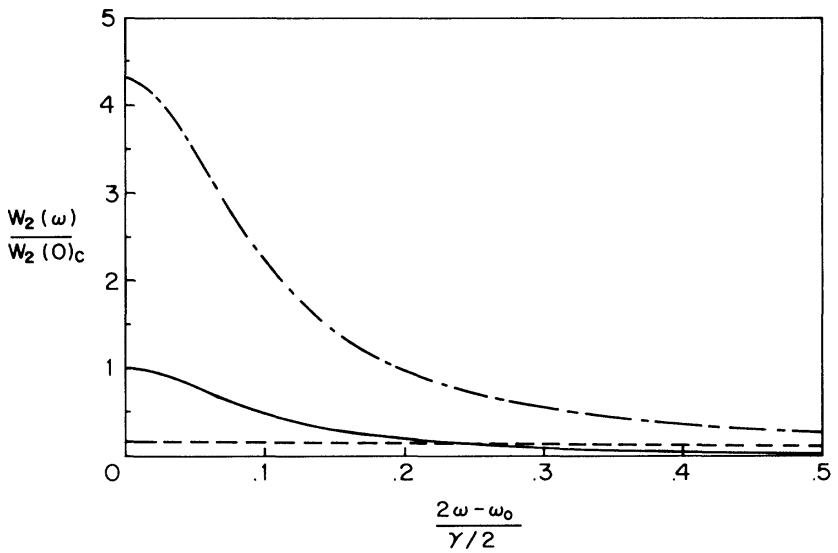

FIG. 1. Absorption spectra for coherent light (solid line), light with squeezed-phase fluctuations (dashed line), and light with squeezed-amplitude fluctuations (dash-dotted line). The parameters chosen are $\Gamma=0.1 \gamma, 2 \epsilon / \gamma=0.2$, and $I_{c} \simeq 5 I_{\mathrm{sv}}$ (see text for details). correlated way may be mentioned. An example has been studied corresponding to a specific assumption for the correlation: namely, $\phi_{c}=\phi_{p} / 2$, where $\phi_{p}$ is the phase of the pump, and the coherent and squeezed-vacuum beams are taken to be superposed outside the OPO cavity, perhaps via a beam splitter. The detailed results for this case will be presented elsewhere. The main difference with the previous case is that now the dominant terms in the spectrum all have the width of the coherent component $\left[4 D_{c}\right.$ replaces $\Gamma / 2$ in $(9)$; cf. Mollow's ${ }^{1}$ results for long- and short-lived atoms]. It is still possible to make the leading terms in the spectrum vanish, albeit for a different value of $I_{c}$; the next largest terms again have a width of $\gamma-2 \epsilon$. The ratio of the (on-resonance) absorption rate for phase-squeezed light to that for amplitude-squeezed light is then of the order of $(\gamma-2 \epsilon) / D_{c}$.

These results are not necessarily restricted to weak fields, since in principle $2 \epsilon$ could be very close to $\gamma$ and hence $I_{\mathrm{sv}}$ [Eq. (4)] might be large. (In practice, however, difficulties would be likely to arise near the threshold of the OPO.)

That the first-order intensity alone may be a poor measure of the response expected in a two-photon process was clearly shown for classical fields by the experiments in Ref. 2. The present work shows that the effect may be much more dramatic for light beams with nonclassical phase or amplitude fluctuations. In particular, Fig. 1 shows that two-photon absorption has the potential to be a very sensitive detector of nonclassical features, magnifying, so to speak, relatively small deviations from "classicity."

I am grateful to the Max-Planck Institut für Quantenoptik (MPQ) for its hospitality at various stages of this work. I am especially indebted to G. Leuchs for suggesting this problem. I have benefited from discussions with many people at MPQ and elsewhere, especially G. Leuchs, W. Schleich, and R. Horowicz.

${ }^{1}$ B. R. Mollow, Phys. Rev. 175, 1555 (1968).

${ }^{2}$ D. S. Elliot, M. W. Hamilton, K. Arnett, and S. J. Smith, 
Phys. Rev. Lett. 53, 439 (1984); Phys. Rev. A 32, 887 (1985).

${ }^{3}$ For a review of the role of fluctuations in multiphoton processes in general, see P. Zoller, in Multiphoton Processes, Springer Series on Atoms and Plasmas Vol. 2, edited by P. Lambropoulos and S. J. Smith (Springer-Verlag, Berlin, 1984), p. 68.

${ }^{4}$ For definitions and general properties of field correlation functions, see R. J. Glauber, in Quantum Optics and Electronics, edited by C. De Witt, A. Blandin, and C. Cohen-Tannoudji (Gordon and Breach, New York, 1965), p. 63.

${ }^{5}$ For revent reviews on theoretical and experimental aspects of squeezed light, see the special issues of the journals $\mathbf{J}$. Mod. Opt. 34, No. 6/7 (1987), and J. Opt. Soc. Am. B 4, No. 10 (1987).

${ }^{6}$ H. P. Yuen, Phys. Rev. A 13, 2226 (1976).

${ }^{7}$ C. M. Caves and B. L. Schumaker, Phys. Rev. A 31, 3068 (1985); B. L. Schumaker and C. M. Caves, Phys. Rev. A 31, 3093 (1985).

${ }^{8}$ C. W. Gardiner, Phys. Rev. Lett. 56, 1917 (1986).

${ }^{9}$ H. J. Carmichael, A. S. Lane, and D. F. Walls, Phys. Rev. Lett. 58, 2539 (1987).

${ }^{10}$ H. Ritsch and P. Zoller, Opt. Commun. 64, 523 (1987); Phys. Rev. Lett. 61, 1097 (1988).

${ }^{11}$ P. R. Rice and H. J. Carmichael, J. Opt. Soc. Am. B 5, 1661 (1988).
${ }^{12}$ J. Gea-Banacloche, Phys. Rev. Lett. 59, 543 (1987).

${ }^{13}$ The work of M. Marte and D. F. Walls, Phys. Rev. A 37, 1235 (1988), and M. A. Marte, H. Ritsch, and D. F. Walls, Phys. Rev. Lett. 61, 1093 (1988), also deals with the effects of squeezed light on laser operation, but like Refs. 8-10 above it assumes that all the modes with which the atoms interact are squeezed.

${ }^{14} \mathrm{G}$. Leuchs, in Frontiers of Nonequilibrium Statistical Physics, edited by G. T. Moore and M. O. Scully, (Plenum, New York, 1986), p. 329.

${ }^{15}$ L. Mandel, Phys. Rev. Lett. 49, 136 (1982).

${ }^{16}$ For a recent and novel approach, see W. Schleich and J. A. Wheeler, J. Opt. Soc. Am. B 4, 1715 (1987).

${ }^{17}$ L.-A. Wu, M. Xiao, and H. J. Kimble, J. Opt. Soc. Am. B 4, 1465 (1987).

${ }^{18}$ M. J. Collet and C. W. Gardiner, Phys. Rev. A 30, 1386 (1984); see also the appendix to M. J. Collet, R. Loudon, and C. W. Gardiner, J. Mod. Opt. 34, 881 (1987).

${ }^{19}$ G. Rempe, H. Walther, and N. Klein, Phys. Rev. Lett. 58, 353 (1987).

${ }^{20}$ M. Brune, J. M. Raimond, P. Goy, L. Davidovich, and S. Haroche, Phys. Rev. Lett. 59, 1899 (1988).

${ }^{21}$ Correlation functions for an OPO with a phase-diffusing pump have been obtained by J. Gea-Banacloche and M. S. Zubairy (to be published). 